\title{
PAHS AND THE CHEMISTRY OF THE ISM
}

\author{
V.M. Bierbaum ${ }^{1}$, V. Le Page ${ }^{1}$ and T.P. Snow ${ }^{2}$
}

\begin{abstract}
This review describes five categories of the reactions of polycyclic aromatic hydrocarbons, including photochemistry, electron attachment/detachment, recombination processes, radical reactions, and ion-neutral chemistry. For each class of reaction, an overview of the studies and their general results are presented, as well as references to the literature. The thermochemistry of PAHs and relevant species is described, including bond dissociation energies, ionization energies, electron affinities, basicities, acidities, and the interrelationships of these quantities. Modeling of the chemistry of PAHs and their ions is discussed for both diffuse and dark clouds. The role of PAH cations in the catalytic formation of molecular hydrogen is considered. Finally, this review concludes with a discussion of current challenges in the chemical characterization of PAHs, and a perspective for future studies.
\end{abstract}

\section{Introduction}

The history of polycyclic aromatic hydrocarbons (PAHs) in the universe has been a fascinating saga, beginning with the ground-breaking proposal of their importance in the cosmos twenty-five years ago (Léger \& Puget 1984; Allamandola et al. 1985). In the intervening years, many scientists have contributed to the unfolding of this story through astronomical observations, laboratory spectroscopy and chemistry, computational characterization, and detailed modeling of astrophysical environments. The integration of these methods and their results has now confirmed the presence of PAHs in many locations throughout the universe. This review will focus on the chemistry of PAHs in the interstellar medium (ISM)(Tielens 2008; Snow \& Bierbaum 2008). Chemistry can be defined as the interactions of elements and compounds with photons, ions, and neutral species, resulting in the formation and dissociation of bonds, the gain and loss of electrons, and the synthesis of the rich chemical structure of the universe. Thus, although chemistry represents only

\footnotetext{
1 Department of Chemistry \& Biochemistry; University of Colorado, Boulder, CO 80309, USA

2 Department of Astrophysical \& Planetary Science; University of Colorado, Boulder, CO 80309, USA
} 
one facet of the complex subject of PAHs in the universe, it is nevertheless an extremely broad and diverse topic, and only a small portion of the literature can be highlighted in this review. Gas phase processes will be discussed at the level of discrete molecules; the chemistry of clusters and grains forms the topics of other manuscripts in this volume.

\section{Reactions of PAHs}

There are several valuable compilations of gas phase reactions: the UMIST Database for Astrochemistry (http://www.udfa.net/); the Ohio State Network (http://www.physics.ohio-state.edu/ eric/research_files/osu_01_2009); KIDA, the Kinetic Database for Astrochemistry (http://kida .obs .u-bordeaux1. $\mathrm{fr} /$ ); and the NIST Chemical Kinetics Database (http://kinetics.nist.gov/ kinetics/index.jsp).

\subsection{Photochemistry}

Photochemistry involves chemical transformations resulting from the interaction of light with matter. Turro et al. (2010) have provided a comprehensive review of the molecular photochemistry of organic molecules that involves a wide array of reactions. This paper will focus on gas phase processes likely to occur in the ISM: the photoionization and dissociation of molecules to form reactive species.

Using naphthalene as an example, photon absorption can result in ionization to form the parent molecular cation; alternatively dissociation can accompany ionization with the loss of hydrogen atom, a hydrogen molecule, or extrusion of a small hydrocarbon such as acetylene.

$$
\begin{aligned}
\mathrm{C}_{10} \mathrm{H}_{8}+\mathrm{h} \nu & \longrightarrow \mathrm{C}_{10} \mathrm{H}_{8}^{+}+e^{-} \\
& \longrightarrow \mathrm{C}_{10} \mathrm{H}_{7}^{+}+\mathrm{H}+e^{-} \\
& \longrightarrow \mathrm{C}_{10} \mathrm{H}_{6}^{+}+\mathrm{H}_{2}+e^{-} \\
& \longrightarrow \mathrm{C}_{8} \mathrm{H}_{6}^{+}+\mathrm{C}_{2} \mathrm{H}_{2}+e^{-}
\end{aligned}
$$

There have been many experimental and theoretical studies of the photochemistry of PAHs and their cations (Verstraete et al. 1990; Jochims et al. 1996; Gotkis et al. 1993; Banisaukas et al. 2004); for example, a comprehensive exploration of the photostability of 33 compounds was carried out using monochromatized synchrotron radiation (Jochims et al. 1999). Results from these various studies indicate that there is a rapidly increasing ionization yield above the ionization energy of the PAH, and rules for determining the quantum yield as a function of wavelength have been proposed. These studies have determined the fragmentation channels and the internal energy required for fragmentation; in general, the loss rates for $\mathrm{H}$ from alkyl groups exceed those of $\mathrm{H}$ from aromatics, which exceed carbon loss as in the form of acetylene.

Photodissociation is being increasingly implemented in Fourier transform ion cyclotron resonance (FT-ICR) experiments to characterize the visible and infrared spectroscopy of PAH cations. The electronic spectra of the pyrene cation, the 
1-methylpyrene cation, the coronene cation and its dehydrogenated derivative have been measured in the PIRENEA experiment using a mid-band OPO laser (Useli-Bacchitta et al. 2010). The infrared spectra of protonated naphthalene, azulene, anthracene, tetracene, pentacene, and coronene have been determined using a free electron laser (Zhao et al. 2009; Knorke et al. 2009).

\subsection{Electron attachment/detachment}

Negative ion formation resulting from low energy (0-15 eV) electron impact of PAHs has been studied in molecular beam experiments (Tobita et al. 1992). The electron detachment cross section is a strong function of the electron affinity of the PAH, which generally increases with size. Thus, for naphthalene, phenanthrene, and triphenylene (which have a negative or only slightly positive electron affinity) no long-lived parent anions $\left(\mathrm{M}^{-}\right)$were observed. The transient negative ion autodetaches before stabilization by photon emission (Tielens 2005). However, for molecules where $\mathrm{EA}>0.5 \mathrm{eV}$, parent negative ions were detected; these compounds include azulene, anthracene, pyrene, acridine, fluoranthene, perylene, and tetracene. For all ten compounds studied, dehydrogenated anions $(\mathrm{M}-\mathrm{H})^{-}$were observed at onset electron energies of about $6 \mathrm{eV}$ (Tobita et al. 1992).

The room temperature rate coefficient for electron attachment to anthracene was measured in a flowing afterglow-Langmuir probe (FALP) experiment to be $1 \times$ $10^{-9} \mathrm{~cm}^{3} \mathrm{~s}^{-1}$ (Canosa et al. 1994). This relatively low value, corresponding to an electron sticking coefficient of only $10^{-4}$, suggests the existence of an energy barrier for this process or the absence of accessible negative ion states for attachment. However, the electron attachment rate coefficient is predicted to have a value about $10^{-6} \mathrm{~cm}^{3} \mathrm{~s}^{-1}$ for PAHs with 30 carbon atoms (Omont 1986; Allamandola et al. 1989). FALP studies indicate that both $\mathrm{C}_{60}$ and $\mathrm{C}_{70}$ efficiently attach electrons above an electron energy of $0.2 \mathrm{eV}$ (Smith \& Spanel 1996).

Photodetachment of PAH anions, with energy resolution of the photoelectrons, has been studied for anthracene and perylene (Schiedt \& Weinkauf 1997a, 1997b), and for chrysene (Tschurl \& Boesl 2006). The electron affinities, vibrational frequencies, and singlet-triplet energy gaps for the neutral molecules can be determined. These relatively limited studies indicate that additional work is needed to fully understand the electron attachment/detachment processes of PAHs.

\subsection{Recombination}

The recombination of molecular cations with electrons or with negative ions results in neutralization and dissociation. A variety of experimental techniques, including merged beams, ion storage rings, afterglow techniques, and shock-tube methods, have contributed a wealth of data on the recombination of electrons with a variety of cations, from simple diatomics to polyatomic species. Larsson \& Orel (2008) have provided an excellent overview of these processes. Many reaction rate constants have been determined, and the temperature dependence and reaction products have been studied for several cations. 
However, studies of ion-electron recombination of PAH cations are relatively sparse. Researchers at Rennes University (Biennier et al. 2006) have recently used the flowing afterglow/photoions method and the FALP technique to measure the room temperature recombination rate coefficients of seven $\mathrm{PAH}$ cations: naphthalene $\left[0.3( \pm 0.1) \times 10^{-6} \mathrm{~cm}^{3} \mathrm{~s}^{-1}\right]$, azulene $\left[1.1( \pm 0.3) \times 10^{-6} \mathrm{~cm}^{3} \mathrm{~s}^{-1}\right]$, acenaphthene $\left[0.5( \pm 0.2) \times 10^{-6} \mathrm{~cm}^{3} \mathrm{~s}^{-1}\right]$, anthracene $\left[2.4( \pm 0.8) \times 10^{-6} \mathrm{~cm}^{3}\right.$ $\left.\mathrm{s}^{-1}\right]$, phenanthrene $\left[1.7( \pm 0.5) \times 10^{-6} \mathrm{~cm}^{3} \mathrm{~s}^{-1}\right]$, fluoranthene $[3.0( \pm 0.9) \times$ $\left.10^{-6} \mathrm{~cm}^{3} \mathrm{~s}^{-1}\right]$, and pyrene $\left[4.1( \pm 1.2) \times 10^{-6} \mathrm{~cm}^{3} \mathrm{~s}^{-1}\right]$. The results indicate an increased reactivity as the number of carbon atoms increases; although the reactions are rapid, they occur below the geometrical limit. Product distributions were not determined; however, these reactions can occur dissociatively to extrude a hydrogen atom or small molecule, or non-dissociatively, especially for larger PAHs.

Hamberg et al. (see elsewhere in this volume) report the temperature dependence of the recombination of the benzene cation and of the protonated benzene cation, using the CRYRING. For $\mathrm{C}_{6} \mathrm{D}_{6}{ }^{+}, \mathrm{k}(\mathrm{T})=1.25 \times 10^{-6}(\mathrm{~T} / 300)^{-0.69} \mathrm{~cm}^{3} \mathrm{~s}^{-1}$, and for $\mathrm{C}_{6} \mathrm{D}_{7}^{+}, \mathrm{k}(\mathrm{T})=2.0 \times 10^{-6}(\mathrm{~T} / 300)^{-0.83} \mathrm{~cm}^{3} \mathrm{~s}^{-1}$. These researchers find that the aromatic ring remains largely intact.

Future studies of the dissociative recombination of larger PAH cations, their temperature dependence, and their reaction products are highly desirable. In addition, while positive ion-negative ion reactions have been studied for simple systems, no measurements exist for PAH ions. Due to the positive electron affinity of most $\mathrm{PAH}$ molecules, these processes will be less exothermic than the corresponding ion-electron recombination, and therefore less dissociation may occur. The development of DESIREE (Schmidt et al. 2008), with two cryogenic ion-storage rings, will make these novel and important studies possible.

\subsection{Radical reactions}

Radical-neutral reactions may be important in the interstellar medium even at low temperatures (Ekern et al. 2007). Recently, Rowe and coworkers have utilized the CRESU apparatus to measure the rate constants for radicals reacting with anthracene between 58-470 K. For OH radical (Goulay et al. 2005), the reaction is rapid and increases as the temperature is lowered: $\mathrm{k}=1.12 \times 10^{-10}$ $(\mathrm{T} / 300)^{-0.46} \mathrm{~cm}^{3} \mathrm{~s}^{-1}$; formation of the adduct is inferred. For $\mathrm{CH}$ radical (Goulay et al. 2006a), the reaction exhibits a slight positive temperature dependence: $\mathrm{k}=$ $(3.32 \pm 1.00) \times 10^{-10}(\mathrm{~T} / 298)^{(0.46 \pm 0.14)} \mathrm{cm}^{3} \mathrm{~s}^{-1}$; however, even at the lowest temperature, the reactivity remains high. The products of the reaction were not determined.

There is an extensive literature describing the synthesis of PAHs under various conditions (Tielens 2008); in some environments the reactions of benzene with carbon-containing radicals provide a probable mechanism for the formation of PAHs (see Cherchneff, this volume). For example, the role of the $\mathrm{CH}_{3}$ radical has been explored, and found to be important in PAH growth (Shukla et al. 2010). The rate coefficient for the reaction of the $\mathrm{C}_{2} \mathrm{H}$ radical with benzene has 
recently been characterized between 105 and $298 \mathrm{~K}$; this value $[\mathrm{k}=(3.28 \pm 1.0) \times$ $\left.10^{-10}(\mathrm{~T} / 298)^{-(0.18 \pm 0.18)} \mathrm{cm}^{3} \mathrm{~s}^{-1}\right]$ indicates that the reaction has no barrier and may play a central role in the formation of large molecules (Goulay \& Leone $2006 \mathrm{~b})$. Additional T-dependent studies of the reactions of radicals with aromatic molecules are clearly warranted.

An especially challenging aspect of the chemistry of PAHs is the existence of isomeric forms and the resulting differences in reactivity and thermodynamics. There is a recent innovative study of Zwier \& co-workers (2010) that explores sitespecific thermochemistry of PAH radicals. These workers have determined bond energies and ionization potentials for the $\mathrm{C}_{10} \mathrm{H}_{9}$ isomeric radicals, 1-hydronaphthyl and 2-hydronaphthyl, from their jet cooled vibronic spectra. These studies reveal the intricacies and the richness of the chemistry of the simplest PAH.

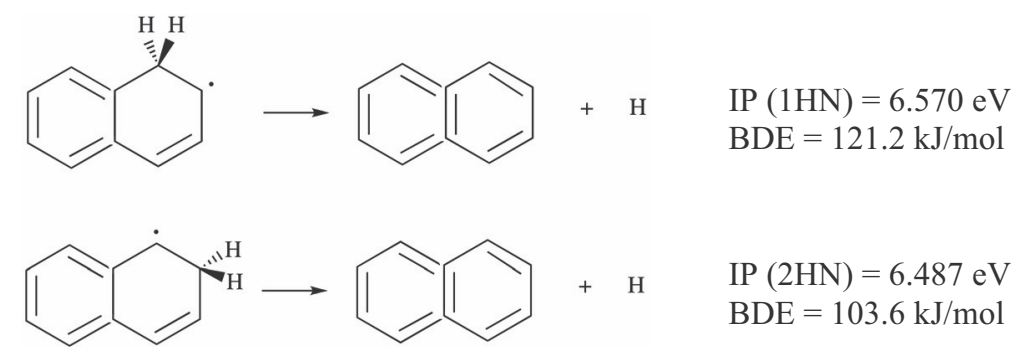

\subsection{Ion-neutral reactions}

The area of gas phase ion chemistry represents an extremely diverse and complex subject of study. Reactions of cations and anions, both simple and complex, can occur with atoms, radicals, and molecules; a multitude of processes are observed, including charge transfer, proton transfer, addition, carbon insertion/loss, hydrogen addition/abstraction, isotope exchange, and associative detachment. Quantitative rate constants, their temperature dependence, and the product distributions are needed for models of interstellar chemistry (Herbst 2001; Smith 2006). This review will briefly consider three categories of reactions, those involving small organic species, fullerenes, and PAHs.

The reactions of small organic ions, $\mathrm{C}_{w} \mathrm{H}_{x} \mathrm{~N}_{y} \mathrm{O}_{z}{ }^{ \pm}$, with a host of neutral reactants have been characterized by many research groups, using a variety of techniques including flow tubes, ICR instruments, and ion traps. There are several excellent reviews of the field (Smith \& Adams 1988; Bohme 2000; Uggerud 2003; Gronert 2005; Nibbering 2006; Gerlich \& Smith 2006). These sequential reactions provide the synthesis of complex species, including PAHs. Although past work has focused on positive ions, anions have recently been detected in molecular clouds; thus, the research in our laboratory is currently centered on negative ion chemistry of small carbon-containing species (Eichelberger et al. 2007; Yang et al. 2010).

Bohme has utilized flow tube mass spectrometry to explore the chemistry of the mono-, di-, and trications of buckminsterfullerene and several substituted cations (Bohme 2009). These ions show remarkable reactivity with a wide variety of 
reagents, which form covalent bonds and thereby generate derivatized fullerenes. Although these processes involve three-body stabilization under the experimental conditions, the radiative processes are likely to be rapid. In analogy, large PAH ions are expected to serve as efficient substrates for addition of many atomic or molecular species.

Unfortunately, experimental studies of the ion chemistry of PAHs are relatively limited. Our work (Snow et al. 1998; Le Page et al. 1999a; Le Page et al. 1999b; Betts et al. 2006) has employed the flowing afterglow-selected ion flow tube (FA-SIFT) technique to explore the chemistry of the parent molecular cations of benzene, naphthalene, pyrene, and coronene $\left(\mathrm{C}_{6} \mathrm{H}_{6}{ }^{+}, \mathrm{C}_{10} \mathrm{H}_{8}{ }^{+}, \mathrm{C}_{16} \mathrm{H}_{10}{ }^{+}\right.$, $\left.\mathrm{C}_{24} \mathrm{H}_{12}{ }^{+}\right)$, the monodehydrogenated cations $\left(\mathrm{C}_{6} \mathrm{H}_{5}{ }^{+}, \mathrm{C}_{10} \mathrm{H}_{7}{ }^{+}, \mathrm{C}_{16} \mathrm{H}_{9}{ }^{+}\right)$, as well as protonated benzene, naphthalene, and pyrene $\left(\mathrm{C}_{6} \mathrm{H}_{7}{ }^{+}, \mathrm{C}_{10} \mathrm{H}_{9}{ }^{+}, \mathrm{C}_{16} \mathrm{H}_{11}{ }^{+}\right)$. For all of these ions, reactions with $\mathrm{H}_{2}, \mathrm{H}, \mathrm{N}$, and $\mathrm{O}$ were characterized; for some ions, reactions with other molecular reagents were also examined $\left(\mathrm{CO}, \mathrm{H}_{2} \mathrm{O}, \mathrm{NH}_{3}, \mathrm{~N}_{2}\right.$, $\left.\mathrm{O}_{2}, \mathrm{NO}, \mathrm{CH}_{3} \mathrm{OH}, \mathrm{CS}_{2},\left(\mathrm{CH}_{3}\right)_{3} \mathrm{~N}\right)$. Consider, for example, the reactions of $\mathrm{C}_{10} \mathrm{H}_{8}{ }^{+}$:

$$
\begin{aligned}
\mathrm{C}_{10} \mathrm{H}_{8}^{+}+\mathrm{H}_{2} & \longrightarrow \text { no reaction } \\
\mathrm{C}_{10} \mathrm{H}_{8}^{+}+\mathrm{H} & \longrightarrow \mathrm{C}_{10} \mathrm{H}_{9}^{+} \\
\mathrm{C}_{10} \mathrm{H}_{8}^{+}+\mathrm{O} & \longrightarrow \mathrm{C}_{10} \mathrm{H}_{8} \mathrm{O}^{+} \\
& \longrightarrow \mathrm{C}_{9} \mathrm{H}_{8}^{+}+\mathrm{CO} \\
\mathrm{C}_{10} \mathrm{H}_{8}^{+}+\mathrm{N} & \longrightarrow \mathrm{C}_{10} \mathrm{H}_{8} \mathrm{~N}^{+} \\
& \longrightarrow \mathrm{C}_{9} \mathrm{H}_{7}^{+}+\mathrm{HCN}
\end{aligned}
$$

The parent molecular cation of naphthalene does not react with molecular hydrogen. However, this radical cation reacts readily with the radical, $\mathrm{H}$-atom, to form protonated naphthalene. In contrast, singlet cations $\left(\mathrm{C}_{6} \mathrm{H}_{5}^{+}, \mathrm{C}_{10} \mathrm{H}_{7}^{+}\right)$have only low reactivity with $\mathrm{H}$ atom, but do react with $\mathrm{H}_{2}$ by addition with moderate rates. Intriguingly, $\mathrm{C}_{16} \mathrm{H}_{9}^{+}$is computed to have a triplet ground state, and this ion reacts efficiently with $\mathrm{H}$ atom but not with $\mathrm{H}_{2}$. Our studies suggest that most $\mathrm{PAH}$ cations will hydrogenate with either atomic or molecular hydrogen.

Although the addition processes in flow tube experiments involve three-body stabilization, it is expected that radiative stabilization will be facile in the ISM. For example, radiative association of molecular hydrogen with $\mathrm{C}_{6} \mathrm{H}_{5}^{+}$and $\mathrm{C}_{10} \mathrm{H}_{7}^{+}$has been observed (Le Page et al. 1999a; Ausloos et al. 1989) at low pressure in ICR instruments. Since the radiative process $\mathrm{C}_{10} \mathrm{H}_{7}^{+}+\mathrm{H}_{2}$ is efficient, the same is likely true for the $\mathrm{C}_{10} \mathrm{H}_{8}^{+}+\mathrm{H}$ reaction; the computed energy content of the intermediate complex $\left[\mathrm{C}_{10} \mathrm{H}_{9}^{+}\right]$is almost identical for these processes, and therefore the complex lifetimes are very similar.

The reactions of $\mathrm{C}_{10} \mathrm{H}_{8}^{+}$with $\mathrm{O}$-atoms and $\mathrm{N}$-atoms illustrated above occur both by association and extrusion of a small stable molecule. This latter channel decreases as the size of the $\mathrm{PAH}$ cation increases; this pathway represents less than $5 \%$ of the reaction for $\mathrm{C}_{16} \mathrm{H}_{10}^{+}$, and is not detectable for $\mathrm{C}_{24} \mathrm{H}_{12}^{+}$. Thus, for large $\mathrm{PAH}$ cations, only addition is observed.

Table 1 summarizes the rate constants for reactions of four cations with atomic reagents. For both $\mathrm{H}$-atoms and $\mathrm{O}$-atoms, the rate constants are large, and the 
Table 1. Comparison of the Reactivity of PAH Parent Molecular Cations with Atomic Reactants. Reaction rate constants are given in units of $\mathrm{cm}^{3} \mathrm{~s}^{-1}$.

\begin{tabular}{|c|c|c|c|}
\hline PAH Cations & $\mathbf{H}$ & $\mathbf{O}$ & $\mathbf{N}$ \\
\hline+ & $2.2 \times 10^{-10}$ & $1.0 \times 10^{-10}$ & $1.2 \times 10^{-10}$ \\
\hline+ & $1.9 \times 10^{-10}$ & $1.0 \times 10^{-10}$ & $2.3 \times 10^{-11}$ \\
\hline
\end{tabular}

reactivity remains high as the $\mathrm{PAH}$ increases in size; this suggests that large $\mathrm{PAH}$ cations, which might exist in the diffuse ISM, will also be reactive with these atomic reagents. In contrast, the reactivity with $\mathrm{N}$-atoms plummets, and becomes negligible for large PAH cations.

\section{Thermochemistry of PAHs}

The chemical reactions of PAHs are intimately related to their thermochemistry. Photochemistry is linked with ionization energy, electron attachment/detachment depends on electron affinity, and radical reactivity reflects bond energies; ionneutral reactions involve these parameters as well as gas phase acidity and basicity values. These quantities are interrelated through thermochemical cycles so that values, which are not easily accessible experimentally, can be computed.

For example, the gas phase acidity of a molecule RH can be measured with flow tube techniques, and the electron affinity of $\mathrm{R}$ can be accurately determined with photoelectron spectroscopy; these values can be combined with the well-known ionization energy of the hydrogen atom to yield the $\mathrm{R}-\mathrm{H}$ bond dissociation energy.

$$
\begin{aligned}
& \mathrm{RH} \longrightarrow \mathrm{R}^{-}+\mathrm{H}^{+} \quad \Delta_{\text {acid }} H(\mathrm{RH}) \\
& \mathrm{R}^{-} \longrightarrow \mathrm{R}+e^{-} \quad \mathrm{EA}(\mathrm{R})
\end{aligned}
$$

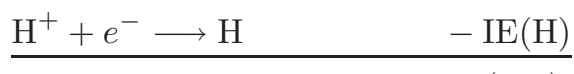

$$
\begin{aligned}
& \mathrm{RH} \longrightarrow \mathrm{R}+\mathrm{H} \quad \mathrm{D}(\mathrm{RH})
\end{aligned}
$$

Figure 1 illustrates the relationship of this negative ion cycle with the proton affinity cycle and the appearance energy cycle (Ervin 2001). A comprehensive compilation of thermodynamic quantities for many PAHs is provided in the NIST Chemistry WebBook (http://webbook.nist.gov/chemistry/) (NIST 2010). 


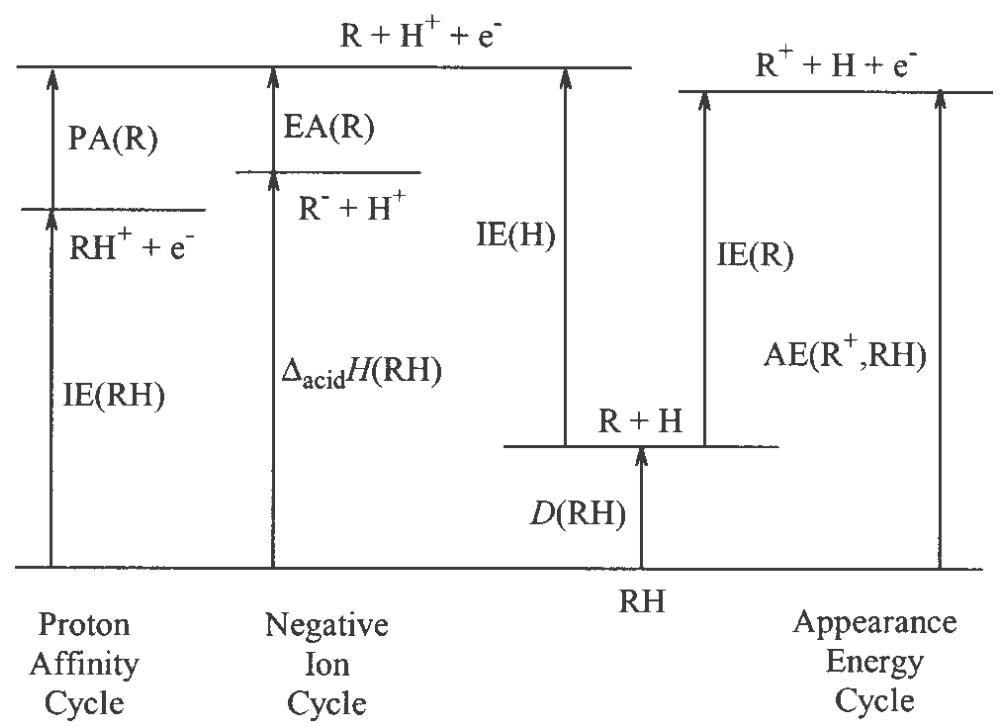

Fig. 1. Thermochemical energy level diagram illustrating the relationship of the proton affinity cycle, the negative ion cycle, and the appearance energy cycle (Ervin 2001).

\section{Modeling of the chemistry of PAHs}

\subsection{Diffuse clouds}

Le Page et al. (2001) have developed a model of the hydrogenation and charge states of PAHs in diffuse clouds; these studies explore which species are likely to survive in environments where the Diffuse Interstellar Bands are formed. The major processes include ionization and photodissociation in the interstellar UV field, recombination of PAH cations, and chemistry of $\mathrm{PAH}$ cations with $\mathrm{H}_{2}$ and $\mathrm{H}$ atoms. The coupled kinetic equations are incorporated into a mathematical framework. Experimental data are employed, and statistical theories are used to estimate properties that are not available from experiments. Other minor processes are considered, including electron attachment, photodetachment, photofragmentation with carbon loss, double ionization, and chemistry with minor species.

Twelve PAH systems were considered, ranging from benzene up to species containing 200 carbon atoms (Le Page et al. 2003). For a standard diffuse cloud, the total $\mathrm{H}$ density is set as $100 \mathrm{~cm}^{-3}$, the ratio of molecular hydrogen to atomic hydrogen density is 0.5 , the scaling of the UV field is unity, the temperature is $100 \mathrm{~K}$, and the ratio of electron density to total $\mathrm{H}$ density is $1.4 \times 10^{-4}$; the effects of varying these parameters were examined. Under most diffuse cloud conditions, the density of the cations exceeds that of neutrals, and the density of anions is low. However, the hydrogenation state strongly depends on the size of the molecule. Small PAHs with fewer than about 15-20 carbon atoms 
are destroyed in most environments. Intermediate-size PAHs with 20-30 carbon atoms are stripped of peripheral hydrogen atoms, but retain their carbon skeleton. Larger PAHs primarily have normal hydrogen coverage, while very large PAHs may be super-hydrogenated. These studies demonstrate that photodissociation and chemical reactions are critical processes governing the behavior of PAHs in the diffuse ISM.

Recently, Montillaud et al. (see elsewhere in this volume) have developed an extended model that includes PAH clusters and PAHs complexed with heavy atoms. Additionally, new reaction rate constants were employed. The first results from this model for coronene appear in this Proceedings volume.

\subsection{Dense clouds}

Wakelam \& Herbst (2008) have recently added PAHs to their gas-phase network of reactions to explore their role in the chemistry of cold dense cores. The processes of radiative electron attachment to form $\mathrm{PAH}^{-}$, recombination between $\mathrm{PAH}$ anions and small cations, and photodetachment of PAH anions by UV radiation were included. A pseudo-time-dependent model, which computes the chemical evolution at a fixed temperature and density, was utilized for three different sets of elemental abundances. The size and abundance of the PAHs were varied to determine the impact of these values on the chemistry. The physical conditions included a temperature of $10 \mathrm{~K}$, a molecular hydrogen density of $10^{4} \mathrm{~cm}^{-3}$, a cosmic-ray ionization rate of $1.3 \times 10^{-17} \mathrm{~s}^{-1}$, and a visual extinction of 10 .

The abundance of PAH cations was found to be at least two orders of magnitude smaller than the neutral or anionic PAH abundances. A dominant effect in the calculations is that PAH anions replace electrons as the dominant carrier of negative charge; this factor reduces the overall ionization fraction because atomic cations recombine more rapidly with $\mathrm{PAH}^{-}$than with electrons. In addition, the abundance of many molecular ions and neutrals are strongly influenced by the presence of PAHs. Inclusion of PAHs in the models significantly improves agreement with observational data for TMC-1, but not for L134N; this result suggests that there may be differences in the PAH properties for the two regions.

\section{PAHs as catalysts in the synthesis of $\mathrm{H}_{2}$}

Bauschlicher (1998) and Hirama and co-workers (2003) have suggested the possible role of $\mathrm{PAH}$ cations in the synthesis of molecular hydrogen:

$$
\begin{aligned}
& \mathrm{PAH}^{+}+\mathrm{H} \longrightarrow \mathrm{PAH}_{+\mathrm{H}}^{+} \\
& \frac{\mathrm{PAH}_{+\mathrm{H}}^{+}+\mathrm{H}}{\mathrm{H}+\mathrm{H}} \longrightarrow \mathrm{PAH}^{+}+\mathrm{H}_{2}
\end{aligned}
$$

$\mathrm{PAH}^{+}$represents any normally hydrogenated $\mathrm{PAH}$ cation, while $\mathrm{PAH}_{+\mathrm{H}}^{+}$represents a cation with an additional hydrogen atom. The first step involves the formation of protonated $\mathrm{PAH}$ by reaction of $\mathrm{H}$-atom with the parent molecular 


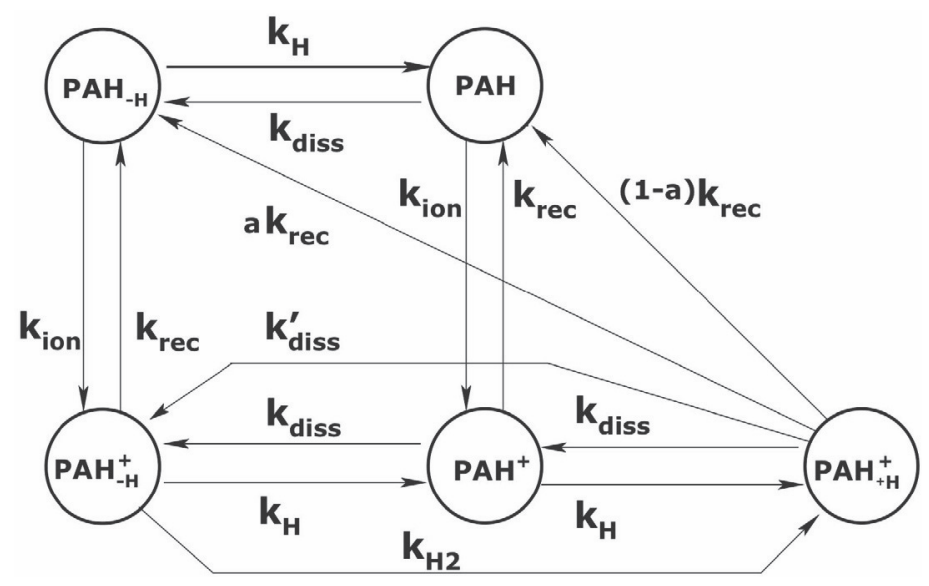

Fig. 2. Chemical mechanism for the catalytic formation of $\mathrm{H}_{2}$ in the interstellar medium (Le Page et al. 2009).

cation; $\mathrm{H}$-atom then abstracts the extra hydrogen to form $\mathrm{H}_{2}$. Since the cations are formed and consumed, the net reaction is formation of a hydrogen molecule from two hydrogen atoms. While we have found the first process to be efficient, our experiments indicate that the reactions of protonated PAHs with $\mathrm{H}$ are slow. We have therefore proposed an alternate model.

Our proposed mechanism consists of four reactions:

$$
\begin{aligned}
& \mathrm{PAH}^{+}+\mathrm{H} \longrightarrow \mathrm{PAH}_{+\mathrm{H}}^{+} \\
& \mathrm{PAH}_{+\mathrm{H}}^{+}+e^{-} \longrightarrow \mathrm{PAH}_{-\mathrm{H}}+\mathrm{H}_{2} \\
& \mathrm{PAH}_{-\mathrm{H}}+\mathrm{H} \longrightarrow \mathrm{PAH}^{\mathrm{PAH}} \longrightarrow \mathrm{PAH}^{+}+e^{-} \\
& \text {Net Reaction } \mathrm{H}+\mathrm{H} \longrightarrow \mathrm{H}_{2}
\end{aligned}
$$

The first step again involves the formation of protonated cations. These species then undergo dissociative recombination with electrons to form the dehydrogenated PAH ( PAH $_{-\mathrm{H}}$ ) and molecular hydrogen; loss of $\mathrm{H}_{2}$ is feasible due to the low binding energy of the two hydrogen atoms bonded to the same carbon. Our model also includes formation of $\mathrm{PAH}+\mathrm{H}$, which is not shown here for simplicity. In the third step, the dehydrogenated radical $\mathrm{PAH}_{-\mathrm{H}}$ combines with hydrogen atom to regenerate the neutral $\mathrm{PAH}$; this molecule is then photoionized to complete the cycle. The complete scheme is summarized in Figure 2. The rate coefficient of $\mathrm{H}_{2}$ production using this process compares well with a widely accepted value of $3 \times 10^{-17} \mathrm{~cm}^{3} \mathrm{~s}^{-1}$ for formation on grains in the diffuse ISM. It is inferred that PAHs can contribute significantly to the formation of molecular hydrogen, especially in environments where high grain temperatures impede the recombination of hydrogen atoms on grain surfaces. 
Recently, there have been additional novel proposals for the production of $\mathrm{H}_{2}$ in the ISM. Vala et al. (2009) have generated protonated 1,2-dihydronaphthalene by electrospray ionization in an FT-ICR, coupled to an IR-tunable free electron laser; infrared multiphoton dissociation studies, complemented by computations, demonstrate the formation of molecular hydrogen. Thrower et al. (see elsewhere in this volume) have used both computational and experimental methods to explore superhydrogenated PAHs; using coronene as an example, these studies indicate efficient routes to $\mathrm{H}_{2}$ formation that may be important in the ISM.

\section{Future perspectives}

There have been major advances in the chemical characterization of PAHs during the last 25 years; nevertheless, there are critical questions remaining for each of the chemical processes discussed in this review. Powerful new developments in experimental methods promise to offer new insights into PAH chemistry in the near future; several of the most crucial areas will be briefly highlighted.

Recombination reactions. There are no experimental data on the neutralization reactions of positive ions with negative ions for PAHs. The dual ion-beam storage ring instrument (DESIREE) (Schmidt et al. 2008) at Stockholm University will enable the measurement of the cross-sections, rate coefficients, and products distributions for these critical reactions.

Reactions at low temperature and pressure. The CRESU technique (Smith 2006) and variable temperature flow tube systems (Smith 1992) have provided experimental data on a variety of ionic processes at low temperature. The recent development of an FT-ICR with cryogenic cooling of the reaction cell (PIRENEA) (Useli-Bacchitta et al. 2010) allows a wide range of ion chemistry experiments under conditions that approach the physical environment of interstellar space $(\mathrm{P} \leq$ $10^{-10}$ mbar, $\mathrm{T}_{\text {trap }} \sim 35 \mathrm{~K}$ ). In addition, two new experimental methods enable the characterization of ion chemistry involving atomic reactants at low energies. These include the innovative atomic beam 22-pole ion trap (Gerlich \& Smith 2006; Luca et al. 2006), which can approach temperatures as low as $10 \mathrm{~K}$, and the novel merged beam apparatus at the Columbia Astrophysics Laboratory (Bruhns et al. 2010).

Characterization of reactants and products. Even the simplest polycyclic aromatic hydrocarbon, naphthalene, possesses distinct chemical sites for protonation, deprotonation, dehydrogenation, addition, and other reactions. The resulting chemical complexity has largely been uncharacterized. New spectroscopic tools (Knorke et al. 2009; Sebree et al. 2010) offer a powerful approach for distinguishing isomeric forms of PAHs and their derivatives, including protonated, hydrogenated, and cyclic versus ring-opened species. Similarly, for many reactions, only the rate constants have been determined, and the identification of products and their branching fractions remains an important area for study.

Reactions of negative ions. In general, gas phase ion chemistry has focused on positive ions, since these species are more readily generated, and PAH cations are the probable form in the diffuse interstellar medium. However, the recognition that 
PAH anions are important in dense clouds (Wakelam \& Herbst 2008) underscores the need for exploring the chemistry of these species. Both electrospray ionization (Fenn et al. 1990) and chemical ionization (Vestal 2001) offer promising approaches for the generation and study of PAH negative ions.

Large PAHs. The low volatility of PAH molecules has largely limited their study in the gas phase to the smaller members of this family. However, modeling studies indicate that moderate and large PAHs are likely to exist in the ISM. Laser Induced Acoustic Desorption (LIAD) (Shea et al. 2007) offers a new, versatile approach for the study of PAHs and a host of other compounds. We are currently coupling a LIAD source to our FA-SIFT instrument to extend our previous work to large, astrophysically relevant PAHs.

In summary, the field of interstellar chemistry of PAHs has a promising and exciting future; it is fascinating to speculate about the marvelous results that will be presented at the 50th anniversary symposium!

The authors are grateful to the National Aeronautics and Space Administration and the National Science Foundation for financial support. We appreciate the many contributions of our colleagues, students, and research associates. We thank Mr. Oscar Martinez Jr. and Mr. Nicholas Demarais for their help in preparation of this manuscript.

\section{References}

Allamandola, L.J., Tielens, A.G.G.M., \& Barker, J.R., 1985, ApJ, 290, L25

Allamandola, L.J., Tielens, A.G.G.M., \& Barker, J.R., 1989, ApJS, 71, 733

Ausloos, P., Lias, S.G., Buckley, T.J., \& Rogers, E.E., 1989, Int. J. Mass Spectrom. Ion Proc., 92, 65

Banisaukas, J., Szczepanski, J., Eyler, J., \& Vala, M., 2004, J. Phys. Chem. A, 108, 3723

Bauschlicher, C.W., 1998, ApJ, 509, L125

Betts, N.B., Stepanovic, M., Snow, T.P., \& Bierbaum, V.M., 2006, ApJ, 651, L129

Biennier, L., Alsayed-Ali, M., Foutel-Richard, A., et al., 2006, Discuss. Faraday Soc., 133,289

Bohme, D.K., 2000, Int. J. Mass Spectrom., 200, 97

Bohme, D.K., 2009, Mass Spectrom. Rev., 28, 672

Bruhns, H., Kreckel, H., Miller, K., et al., 2010, Rev. Sci. Instr., 81, 013112

Canosa, A., Parent, D.C., Pasquerault, D., et al., 1994, Chem. Phys. Lett., 228, 26

Eichelberger, B.R., Snow, T.P., Barckholtz, C., \& Bierbaum, V.M., 2007, ApJ, 667, 1283

Ekern, S., Gomez, L., Szczepanski, J., \& Vala, M., 2007, Chem. Phys., 331, 219

Ervin, K.M., 2001, Chem. Rev., 101, 391

Fenn, J.B., Mann, M., Meng, C.K., Wong, S.F., \& Whitehouse, C.M., 1990, Mass Spectrom. Rev., 9, 37

Gerlich, D., \& Smith, M., 2006, Phys. Scr., 73, C25

Gotkis, Y., Oleinikova, M., Naor, M., \& Lifshitz, C., 1993, J. Phys. Chem., 97, 12282

Goulay, F., Rebrion-Rowe, C., Garrec, J.L.L., et al., 2005, J. Chem. Phys., 122, 104308

Goulay, F., Rebrion-Rowe, C., Biennier, L., et al., 2006a, J. Phys. Chem. A, 110, 3132 
Goulay, F., \& Leone, S.R., 2006b, J. Phys. Chem. A, 110, 1875

Gronert, S., 2005, Mass Spectrom. Rev., 24, 100

Herbst, E., 2001, Chem. Soc. Rev., 30, 168

Hirama, M., Ishida, T., \& Aihara, J.-I., 2003, J. Comput. Chem., 24, 1378

Jochims, H.W., Baumgartel, H., \& Leach, S., 1996, A\&A, 314, 1003

Jochims, H.W., Baumgartel, H., \& Leach, S., 1999, ApJ, 512, 500

Knorke, H., Langer, J., Oomens, J., \& Dopfer, O., 2009, ApJ, 706, L66

Larsson, M., \& Orel, A.E., 2008, Dissociative Recombination of Molecular Ions (Cambridge University Press: Cambridge)

Le Page, V., Keheyan, Y., Snow, T.P., \& Bierbaum, V.M., 1999a, J. Am. Chem. Soc., 121, 9435

Le Page, V., Keheyan, Y., Snow, T.P., \& Bierbaum, V.M., 1999b, Int. J. Mass Spectrom., 185/186/187, 949

Le Page, V., Snow, T.P., \& Bierbaum, V.M., 2001, ApJS, 132, 233

Le Page, V., Snow, T.P., \& Bierbaum, V.M., 2003, ApJ, 584, 316

Le Page, V., Snow, T.P., \& Bierbaum, V.M., 2009, ApJ, 704, 274

Léger, A., \& Puget, J.L., 1984, A\&A, 137, L5

Linstrom, P.J., \& Mallard, W.G. (eds.), NIST Chemistry WebBook, NIST Standard Reference Database Number 69, National Institute of Standards and Technology, Gaithersburg, MD 20899, http://webbook.nist.gov

Luca, A., Borodi, G., \& Gerlich, D., 2006, in "Interactions of Ions with Hydrogen Atoms. In Progress report in XXIV ICPEAC 2005", ed. Colavecchia, F.D., Fainstein, P.D., Fiol, J., Lima, M.A.P., Miraglia, J.E., Montenegro, E.C.. Rivarola, R.D. (Rosario, Argentina), 29

Nibbering, N.M.M., 2006, Mass Spectrom. Rev., 25, 962

Omont, A., 1986, A\&A, 164, 159

Schiedt, J., \& Weinkauf, R., 1997a, Chem. Phys. Lett., 266, 201

Schiedt, J., \& Weinkauf, R., 1997b, Chem. Phys. Lett., 274, 18

Schmidt, H.T., Johansson, H.A.B., Thomas, R.D., et al., 2008, Int. J. Astrobiology, 7, 205

Sebree, J.A., Kislov, V.V., Mebel, A.M., \& Zwier, T.S., 2010, J. Phys. Chem. A, 114, 6255

Shea, R.C., Habicht, S.C., Vaughn, W.E., \& Kenttamaa, H.I., 2007, Anal. Chem., 79, 2688

Shukla, B., Miyoshi, A., \& Koshi, M., 2010, J. Am. Soc. Mass Spectrom., 21, 534

Smith, D., \& Adams, N.G., 1988, Adv. At. Mol. Phys., 24, 1

Smith, D., 1992, Chem. Rev., 92, 1473

Smith, D., \& Spanel, P., 1996, J. Phys. B: At. Mol. Opt. Phys., 29, 5199

Smith, I.W.M., 2006, Angew. Chem. Int. Ed., 45, 2842

Snow, T.P., Page, V.L., Keheyan, Y., \& Bierbaum, V.M., 1998, Nature, 391, 259

Snow, T.P., \& Bierbaum, V.M., 2008, Annu. Rev. Anal. Chem., 1, 229

Tielens, A.G.G.M., 2005, "The Physics and Chemistry of the Interstellar Medium" (Cambridge Univ. Press: Cambridge, UK)

Tielens, A.G.G.M., 2008, ARA\&A, 46, 289 
Tobita, S., Meinke, M., Illenberger, E., et al., 1992, Chem. Phys., 161, 501

Tschurl, M., \& Boesl, U., 2006, Int. J. Mass Spectrom., 249, 364

Turro, N.J., Ramamurthy, V., \& Scaiano, J.C., 2010, "Modern Molecular Photochemistry of Organic Molecules" (University Science Books: Sausalito, CA)

Uggerud, E., 2003, Top. Curr. Chem., 225, 3

Useli-Bacchitta, F., Bonnamy, A., Mulas, G., et al., 2010, Chem. Phys., 371, 16

Vala, M., Szczepanski, J., Oomens, J., \& Steill, J.D., 2009, J. Am. Chem. Soc., 131, 5784

Verstraete, L., Léger, A., d'Hendecourt, L., Dutuit, O., \& Defourneau, D., 1990, Astron. Astrophys., 237, 436

Vestal, M.L., 2001, Chem. Rev., 101, 361

Wakelam, V., \& Herbst, E., 2008, ApJ, 680, 371

Yang, Z., Eichelberger, B., Carpenter, M., et al., 2010, submitted

Zhao, D., Langer, J., Oomens, J., \& Dopfer, O., 2009, J. Chem. Phys., 131, 184307 